PROCEEDINGS OF THE

AMERICAN MATHEMATICAL SOCIETY

Volume 125, Number 8, August 1997, Pages 2293-2305

S 0002-9939(97)03867-7

\title{
THE MODULAR INEQUALITIES FOR A CLASS OF CONVOLUTION OPERATORS ON MONOTONE FUNCTIONS
}

\author{
JIM QILE SUN
}

(Communicated by Palle E. T. Jorgensen)

AbStRACT. This paper is devoted to the study of modular inequality

$$
\Phi_{2}^{-1}\left(\int_{0}^{+\infty} \Phi_{2}(a(x) K f(x)) w(x) d x\right) \leq \Phi_{1}^{-1}\left(\int_{0}^{+\infty} \Phi_{1}(C f(x)) v(x) d x\right)
$$

where $\Phi_{1} \ll \Phi_{2}$ and $K$ is a class of Volterra convolution operators restricted to the monotone functions. When $\Phi_{1}(x)=x^{p} / p, \Phi_{2}(x)=x^{q} / q$ with $1<p \leq$ $q<+\infty$ and the kernel $k(x) \equiv 1$, our results will extend those for the Hardy operator on monotone functions on Lebesgue spaces.

\section{INTRODUCTION}

Let $K$ be a class of Volterra convolution operators given by

$$
K f(x)=\int_{0}^{x} k(x-t) f(t) d t
$$

where the kernel $k$ satisfies the following conditions:

(a) $k(x) \geq 0$ is nondecreasing on $(0,+\infty)$;

(b) $k(x+y) \leq D(k(x)+k(y))$ for all $x, y \in(0,+\infty)$.

Consider the problem of characterizing the weights $a, w, v$ for which the modular inequality of the form

$$
\Phi_{2}^{-1}\left(\int_{0}^{+\infty} \Phi_{2}(a K f(x)) w(x) d x\right) \leq \Phi_{1}^{-1}\left(\int_{0}^{+\infty} \Phi_{1}(C f(x)) v(x) d x\right)
$$

holds for all nonnegative monotone functions $f$. This sort of problem on Lebesgue spaces for the Hardy operator has been widely studied in [ES], [VS] and [HS]. In this paper, we will characterize the weights $w, v$ for the above modular inequality when $\Phi_{1} \ll \Phi_{2}$ and the operator $K$ is restricted to the monotone functions. The results are even new on Orlicz spaces when $K$ is the Hardy operator.

It is worth mentioning that when $\Phi_{1} \in \Delta_{2}$, the above inequality is equivalent to

$$
\Phi_{2}^{-1}\left(\int_{0}^{+\infty} \Phi_{2}(a K f) w\right) \leq C \Phi_{1}^{-1}\left(\int_{0}^{+\infty} \Phi_{1}(f) v\right) .
$$

We begin with a brief summary of the notations on the Orlicz space setting.

Received by the editors July 10, 1995 and, in revised form, January 30, 1996.

1991 Mathematics Subject Classification. Primary 26D15, 42B25.

(C)1997 American Mathematical Society 
An N-function $\Phi$ is a continuous Young's function such that

$$
\Phi(t)=\int_{0}^{t} \phi
$$

where $\phi(t)$ is a nondecreasing right continuous function defined on $[0,+\infty)$ with $\phi(0+)=0, \phi(+\infty)=+\infty$. Let $\phi^{-1}$ be the right continuous inverse function of $\phi ;$ then

$$
\Psi(t)=\int_{0}^{t} \phi^{-1}
$$

is called the complementary function of $\Phi(t)$.

Definition 1. a) An N-function $\Phi$ is said to satisfy the $\Delta_{2}$ condition (we write $\left.\Phi \in \Delta_{2}\right)$ if there is a constant $B>0$, such that

$$
\Phi(2 t) \leq B \Phi(t) \quad \forall t>0 .
$$

b) We write $\Phi_{1} \ll \Phi_{2}$ if there is a constant $L_{0}>0$, such that

$$
\sum \Phi_{2} \circ \Phi_{1}^{-1}\left(a_{i}\right) \leq L_{0} \Phi_{2} \circ \Phi_{1}^{-1}\left(\sum a_{i}\right)
$$

holds for every sequence $\left\{a_{i}\right\}$ with $a_{i} \geq 0$.

c) Let $w$ be a nonnegative, measurable weight function and $\Phi$ an N-function. The Orlicz space $L_{\Phi}(w)$ consists of all nonnegative measurable functions $f$ (modulo the equivalence relation almost everywhere) such that

$$
\|f\|_{\Phi(w)}=\inf \left\{\lambda>0: \int_{0}^{+\infty} \Phi(f / \lambda) w \leq 1\right\}
$$

is finite. We call $\|\cdot\|_{\Phi(w)}$ the Luxemburg norm.

For more standard theory of Orlicz spaces, see $[\mathrm{KR}]$ and $[\mathrm{RR}]$.

For the proofs of our coming theorems, we need the following special results from Chapter 2 of [JS].

Proposition 1. Let $\Phi_{1} \ll \Phi_{2}$, and let $a, b, w, v$ be weight functions. Then there exists a constant $C$ such that

$$
\Phi_{2}^{-1}\left(\int_{0}^{+\infty} \Phi_{2}(a K f) w\right) \leq \Phi_{1}^{-1}\left(\int_{0}^{+\infty} \Phi_{1}(C b f) v\right)
$$

holds for all nonnegative functions $f$ if and only if there exists a constant B such that both

$$
\Phi_{2}^{-1}\left(\int_{r}^{+\infty} \Phi_{2}\left(\frac{a(x)}{B}\left\|\frac{k(r-\cdot) \chi_{(0, r)}}{\varepsilon v b}\right\|_{\Psi_{1}(\varepsilon v)}\right) w(x) d x\right) \leq \Phi_{1}^{-1}\left(\frac{1}{\varepsilon}\right)
$$

and

$$
\Phi_{2}^{-1}\left(\int_{r}^{+\infty} \Phi_{2}\left(\frac{a(x)}{B}\left\|\frac{\chi_{(0, r)}}{\varepsilon v b}\right\|_{\Psi_{1}(\varepsilon v)} k(x-r)\right) w(x) d x\right) \leq \Phi_{1}^{-1}\left(\frac{1}{\varepsilon}\right)
$$

hold for all $\varepsilon, r>0$.

We define the dual operator of

$$
K f(x)=\int_{0}^{x} k(x-t) f(t) d t \quad \text { by } \quad K^{\star} g(t)=\int_{t}^{+\infty} k(x-t) g(x) d x .
$$


For the operator $K^{\star}$, we have

Proposition 2. Let $\Phi_{1} \ll \Phi_{2}$. Then there exists a constant $C$ such that

$$
\Phi_{2}^{-1}\left(\int_{0}^{+\infty} \Phi_{2}\left(a K^{\star} g\right) w\right) \leq \Phi_{1}^{-1}\left(\int_{0}^{+\infty} \Phi_{1}(C b g) v\right)
$$

holds for all nonnegative measurable functions $g$ if and only if there exists a constant $B$ such that both

$$
\Phi_{2}^{-1}\left(\int_{0}^{r} \Phi_{2}\left(\frac{a(t)}{B}\left\|\frac{k(\cdot, r) \chi_{(r,+\infty)}}{\varepsilon v b}\right\|_{\Psi_{1}(\varepsilon v)}\right) w(t) d t\right) \leq \Phi_{1}^{-1}\left(\frac{1}{\varepsilon}\right)
$$

and

$$
\Phi_{2}^{-1}\left(\int_{0}^{r} \Phi_{2}\left(\frac{a(t)}{B}\left\|\frac{\chi_{(r,+\infty)}}{\varepsilon v b}\right\|_{\Psi_{1}(\varepsilon v)} k(r, t)\right) w(t) d t\right) \leq \Phi_{1}^{-1}\left(\frac{1}{\varepsilon}\right)
$$

hold for any $\varepsilon, r>0$.

Throughout this paper, we use $C$ to denote constants which may be different at different places, although in some instances we write $C_{1}, C_{2}, \cdots$ to indicate different constants. Also, we write

$$
V(x)=\int_{0}^{x} v \text { and } \quad V^{\star}(x)=\int_{x}^{+\infty} v .
$$

\section{The MAIN RESUlts}

We have the following main results.

Theorem 1. Let both $\Phi_{1}$ and its complementary function $\Psi_{1}$ satisfy the $\Delta_{2}$ condition and suppose that $\Phi_{1} \ll \Phi_{2}$. If $K$ is a class of Volterra convolution operators, then

$$
\Phi_{2}^{-1}\left(\int_{0}^{+\infty} \Phi_{2}(a K f) w\right) \leq \Phi_{1}^{-1}\left(\int_{0}^{+\infty} \Phi_{1}(C f) v\right)
$$

holds for all nonnegative, nonincreasing functions $f$ if and only if there is a constant $B$ such that all of the following inequalities hold for all $\varepsilon, r>0$ :

$$
\begin{gathered}
\Phi_{2}^{-1}\left(\int_{0}^{r} \Phi_{2}\left(\varepsilon a(x) \int_{0}^{x} k(y) d y\right) w(x) d x\right) \leq \Phi_{1}^{-1}\left(\Phi_{1}(B \varepsilon) V(r)\right) ; \\
\Phi_{2}^{-1}\left(\int_{r}^{+\infty} \Phi_{2}\left(\frac{a(x)}{B}\left\|\frac{g_{0} k(r-\cdot) \chi_{(0, r)}}{\varepsilon V}\right\|_{\Psi_{1}(\varepsilon v)}\right) w(x) d x\right) \leq \Phi_{1}^{-1}\left(\frac{1}{\varepsilon}\right) ; \\
\Phi_{2}^{-1}\left(\int_{r}^{+\infty} \Phi_{2}\left(\frac{a(x)}{B}\left\|\frac{g_{0} \chi_{(0, r)}}{\varepsilon V}\right\|_{\Psi_{1}(\varepsilon v)} k(x-r)\right) w(x) d x\right) \leq \Phi_{1}^{-1}\left(\frac{1}{\varepsilon}\right) ; \\
\Phi_{2}^{-1}\left(\int_{r}^{+\infty} \Phi_{2}\left(\frac{a(x)}{B}\left\|\frac{g_{1} \chi_{(0, r)}}{\varepsilon V}\right\|_{\Psi_{1}(\varepsilon v)}\right) w(x) d x\right) \leq \Phi_{1}^{-1}\left(\frac{1}{\varepsilon}\right) .
\end{gathered}
$$

Here $g_{0}(s)=s$ and $g_{1}(s)=\int_{0}^{s} k(t) d t$. 
For the next theorem, we need the following additional condition on the kernel $k$. There is a constant $D^{\prime}$ such that

$$
\widetilde{k}(x+y) \leq D^{\prime}(\widetilde{k}(x)+\widetilde{k}(y)),
$$

where $\widetilde{k}(x)=\int_{0}^{x} k$.

Theorem 2. Let both $\Phi_{1}$ and its complementary function $\Psi_{1}$ satisfy the $\Delta_{2}$ condition and suppose $\Phi_{1} \ll \Phi_{2}$. If $K$ is a Volterra convolution operator whose kernel $k$ satisfies the above condition, then (1) holds for all nonnegative, nondecreasing functions $f$ if and only if there is a constant $B$ such that

$$
\Phi_{2}^{-1}\left(\int_{r}^{+\infty} \Phi_{2}\left(\frac{a(x)}{B}\left\|\frac{\widetilde{k}(r-\cdot) \chi_{(0, r)}}{\varepsilon V^{\star}}\right\|_{\Psi_{1}(\varepsilon v)}\right) w(x) d x\right) \leq \Phi_{1}^{-1}\left(\frac{1}{\varepsilon}\right)
$$

and

$$
\Phi_{2}^{-1}\left(\int_{r}^{+\infty} \Phi_{2}\left(\frac{a(x)}{B} \Phi_{1}^{-1}\left(\frac{1}{\varepsilon V^{\star}(r)}\right) \widetilde{k}(x-r)\right) w(x) d x\right) \leq \Phi_{1}^{-1}\left(\frac{1}{\varepsilon}\right)
$$

hold for any $\varepsilon, r>0$.

There are similar conclusions for the dual operator $K^{\star}$; we leave it to the reader as an easy exercise. In Theorems 1 and 2 , when $k(x) \equiv 1, K$ is the Hardy operator $H f(x)=\int_{0}^{x} f$ and $\int_{0}^{s} k=s$. Then we have

Corollary 1. Let both $\Phi_{1}$ and its complementary function $\Psi_{1}$ satisfy the $\Delta_{2}$ condition, $\Phi_{1} \ll \Phi_{2}$. Then

$$
\Phi_{2}^{-1}\left(\int_{0}^{+\infty} \Phi_{2}(a H f) w\right) \leq \Phi_{1}^{-1}\left(\int_{0}^{+\infty} \Phi_{1}(C f) v\right)
$$

holds for all nonnegative nonincreasing functions $f$ if and only if there exists a constant B such that both

$$
\Phi_{2}^{-1}\left(\int_{0}^{r} \Phi_{2}(\varepsilon x a(x)) w(x) d x\right) \leq \Phi_{1}^{-1}\left(\Phi_{1}(B \varepsilon) V(r)\right)
$$

and

$$
\Phi_{2}^{-1}\left(\int_{r}^{+\infty} \Phi_{2}\left(\frac{a(x)}{B}\left\|\frac{g_{0} \chi_{(0, r)}}{\varepsilon V}\right\|_{\Psi_{1}(\varepsilon v)}\right) w(x) d x\right) \leq \Phi_{1}^{-1}\left(\frac{1}{\varepsilon}\right)
$$

hold for all $\varepsilon, r>0$.

There is a similar result when $H$ is restricted to nondecreasing functions by our Theorem 2. We omit the details.

When $a(x)=1 / x, a(x) H f(x)=\frac{1}{x} \int_{0}^{x} f$, it is the Hardy averaging operator. Moreover, if $\Phi_{1}(x)=x^{p} / p, \Phi_{2}(x)=x^{q} / q$ with $1<p \leq q<+\infty$, then Corollary 1 recovers some of the results in [ES], [VS] and [HS].

\section{Proof of Theorem 1}

We need the following results from Chapter 3 of [JS].

Lemma 1. Let $\Phi_{1} \ll \Phi_{2}$; then

$$
\Phi_{2}^{-1}\left(\int_{0}^{+\infty} \Phi_{2}(a(x) f(x)) w(x) d x\right) \leq \Phi_{1}^{-1}\left(\int_{0}^{+\infty} \Phi_{1}(C f(x)) v(x) d x\right)
$$


holds for all nonincreasing $f$ if and only if there is a constant $B$ such that

$$
\Phi_{2}^{-1}\left(\int_{0}^{r} \Phi_{2}(\varepsilon a(x)) w(x) d x\right) \leq \Phi_{1}^{-1}\left(\Phi_{1}(B \varepsilon) V(r)\right)
$$

holds for any $\varepsilon, r>0$.

Lemma 2. Suppose $f$ is a nonnegative, nondecreasing function on $(0,+\infty)$. Then there exists a sequence $\left\{h_{n}\right\}$, of nonnegative functions, each compactly supported in $(0,+\infty)$, such that for almost each $x>0, \int_{0}^{x} h_{n}$ increases to $f(x)$ as $n \rightarrow+\infty$.

Also if $f$ is a nonnegative, nonincreasing function on $(0,+\infty)$, then there exists a sequence $\left\{h_{n}\right\}$, of nonnegative functions, each compactly supported in $(0,+\infty)$, such that for almost each $x>0, \int_{x}^{+\infty} h_{n}$ increases to $f(x)$ as $n \rightarrow+\infty$.

For the proofs of these two lemmas, see [JS], pages 39-41 and page 48 respectively.

We also need the following lemmas from Chapter 4 of [JS].

Lemma 3. Let $\Phi$ and its complementary function $\Psi$ be in $\Delta_{2}$. Then

$$
\Phi^{-1}\left(\int_{0}^{+\infty} \Phi\left(\frac{\int_{0}^{x} f(t) v(t) d t}{V(x)}\right) v(x) d x\right) \leq \Phi^{-1}\left(\int_{0}^{+\infty} \Phi(C f(x)) v(x) d x\right)
$$

holds for any $f \geq 0$ with the constant $C$ independent of $f$.

The proof of the lemma can be found either in [HK] or [JS], pages 60-61. It can also be deduced from Proposition 1 with $k(x) \equiv 1$.

Lemma 4. Let $\Phi$ and its complementary function $\Psi$ be in $\Delta_{2}$. Then

$$
\Phi^{-1}\left(\int_{0}^{+\infty} \Phi\left(\int_{x}^{+\infty} \frac{f(t) v(t)}{V(t)} d t\right) v(x) d x\right) \leq \Phi^{-1}\left(\int_{0}^{+\infty} \Phi(C f(x)) v(x) d x\right)
$$

holds for any $f \geq 0$ with the constant $C$ independent of $f$.

Proof. By Proposition 2, it is easy to see that the inequality in the lemma is valid provided that there exists a constant $B$ such that

$$
\Phi^{-1}\left(\int_{0}^{r} \Phi\left(\frac{1}{B}\left\|\frac{\chi_{(r,+\infty)}}{\varepsilon V}\right\|_{\Psi_{1}(\varepsilon v)}\right) v(t) d t\right) \leq \Phi^{-1}\left(\frac{1}{\varepsilon}\right)
$$

holds for all $\varepsilon, r>0$. This can be proved by the similar technique used in [HK].

3.1 Proof of sufficiency. Lemma 2 shows that without loss of generality, we may suppose $f(x)=\int_{x}^{+\infty} h$, with $h$ compactly supported in $(0,+\infty)$ and $\int_{0}^{+\infty} \Phi_{1}(f) v<$ $+\infty$. We have

$$
\begin{aligned}
K f(x) & =\int_{0}^{x} k(x-y)\left(\int_{y}^{+\infty} h(t) d t\right) d y \\
& =\int_{0}^{x}\left(\int_{0}^{s} k(x-y) d y\right) h(s) d s+f(x) \int_{0}^{x} k(y) d y \\
& \equiv K_{1}+K_{2} .
\end{aligned}
$$

For $K_{2}$, apply Lemma 1 with $a(x)$ replaced by $a(x) \int_{0}^{x} k(t) d t$ and use condition (2) to get

$$
\begin{gathered}
\Phi_{2}^{-1}\left(\int_{0}^{+\infty} \Phi_{2}\left(a(x) \int_{0}^{x} k(t) d t f(x)\right) w(x) d x\right) \\
\leq \Phi_{1}^{-1}\left(\int_{0}^{+\infty} \Phi_{1}\left(C_{1} f(x)\right) v(x) d x\right) .
\end{gathered}
$$


Since $V(s)^{-1}=V(x)^{-1}+\int_{s}^{x} V(t)^{-2} v(t) d t$ and $\int_{0}^{x} h(s) V(s) d s \leq \int_{0}^{x} f(s) v(s) d s$, we have

$$
\begin{aligned}
K_{1} & =\int_{0}^{x}\left(\int_{0}^{s} k(x-y) d y\right) h(s) V(s)\left(V(x)^{-1}+\int_{s}^{x} V(t)^{-2} v(t) d t\right) d s \\
& \leq V(x)^{-1} \int_{0}^{x} k(x-y) \int_{y}^{x} h(s) V(s) d s d y \\
& +\int_{0}^{x}\left(\int_{0}^{s} k(x-y) d y\right) h(s) V(s) \int_{s}^{x} V(t)^{-2} v(t) d t d s \\
& \leq \int_{0}^{x} k(x-y) d y \frac{\int_{0}^{x} f(t) v(t) d t}{V(x)} \\
& +\int_{0}^{x} \int_{0}^{t}\left(\int_{0}^{s} k(x-y) d y\right) h(s) V(s) d s V(t)^{-2} v(t) d t \\
& \leq \int_{0}^{x} k(x-y) d y \frac{\int_{0}^{x} f(t) v(t) d t}{V(x)} \\
& +\int_{0}^{x}\left(\int_{0}^{t} k(x-y) d y\right) \frac{\int_{0}^{t} f(s) v(s) d s}{V(t)^{2}} v(t) d t \\
& \equiv K_{3}+K_{4},
\end{aligned}
$$

where we have used the property (a) of the kernel $k$ for the last inequality.

Since $\int_{0}^{x} f(s) v(s) d s / V(x)$ is also a nonincreasing function, Lemma 1 again shows that for $K_{3}$,

$$
\begin{aligned}
& \Phi_{2}^{-1}\left(\int_{0}^{+\infty} \Phi_{2}\left(a(x) \int_{0}^{x} k(y) d y \frac{\int_{0}^{x} f(s) v(s) d s}{V(x)}\right) w(x) d x\right) \\
& \leq \Phi_{1}^{-1}\left(\int_{0}^{+\infty} \Phi_{1}\left(C_{1} \frac{\int_{0}^{x} f(s) v(s) d s}{V(x)}\right) v(x) d x\right) .
\end{aligned}
$$

Now, according to Lemma 3, we see that

$$
\Phi_{1}^{-1}\left(\int_{0}^{+\infty} \Phi_{1}\left(\frac{\int_{0}^{x} f v}{V(x)}\right) v(x) d x\right) \leq \Phi_{1}^{-1}\left(\int_{0}^{+\infty} \Phi_{1}\left(C_{2} f(x)\right) v(x) d x\right)
$$

which completes the estimate for $K_{3}$.

For $K_{4}$, notice that

$$
\int_{0}^{t} k(x-y) d y \leq D\left(k(x-t) t+\int_{0}^{t} k(y) d y\right), \quad \text { for } 0<y<t<x .
$$

We have

$$
\begin{aligned}
K_{4} & \leq D\left(\int_{0}^{x} k(x-t) \frac{t \int_{0}^{t} f(s) v(s) d s}{V(t)^{2}} v(t) d t+\int_{0}^{x}\left(\int_{0}^{t} k(y) d y\right) \frac{\int_{0}^{t} f v}{V(t)^{2}} v(t) d t\right) \\
& \equiv D\left(K_{5}+K_{6}\right) .
\end{aligned}
$$


For $K_{5}$, consider the inequality

$$
\begin{aligned}
\Phi_{2}^{-1} & \left(\int_{0}^{+\infty} \Phi_{2}\left(a(x) \int_{0}^{x} k(x-s) g(s) d s\right) w(x) d x\right) \\
& \leq \Phi_{1}^{-1}\left(\int_{0}^{+\infty} \Phi_{1}\left(C_{3} g(t) b(t)\right) v(t) d t\right),
\end{aligned}
$$

where $g(t)=t v(t) V(t)^{-2} \int_{0}^{t} f v$ and $b(t)=V(t) /(t v(t))$. Proposition 1 shows that (8) holds for any $g \geq 0$ provided conditions (3) and (4) hold.

Since $g(t) b(t)=\int_{0}^{t} f(s) v(s) d s / V(t)$, Lemma 3 again shows that the right hand side of $(8)$ is bounded by

$$
\Phi_{1}^{-1}\left(\int_{0}^{+\infty} \Phi_{1}(C f(x)) v(x) d x\right) .
$$

For $K_{6}$, consider the inequality

$$
\begin{aligned}
\Phi_{2}^{-1} & \left(\int_{0}^{+\infty} \Phi_{2}\left(a(x) \int_{0}^{x} G(t) d t\right) w(x) d x\right) \\
& \leq \Phi_{1}^{-1}\left(\int_{0}^{+\infty} \Phi_{1}\left(C_{4} G(t) b(t)\right) v(t) d t\right),
\end{aligned}
$$

where $G(t)=\left(v(t) \int_{0}^{t} k(y) d y \int_{0}^{t} f v\right) / V(t)^{2}$ and $b(t)=V(t) /\left(v(t) \int_{0}^{t} k(y) d y\right)$. Applying Proposition 1 once more for the Hardy operator, we see that (9) holds for $G(t) \geq 0$ provided condition (5) holds.

Combining all of the above and using the convexity of $\Phi_{1}$, the result follows.

Sufficiency is proved.

3.2 Proof of necessity. Since $K f(x) \geq\left(\int_{0}^{x} k(y) d y\right) f(x)$ for any nonincreasing function $f$, we may obtain condition (2) by taking $f=\varepsilon \chi_{(0, r)}$ for each $\varepsilon, r>0$.

Noticing the Orlicz norm dominates the Luxemburg norm (see [KR], page 80), we have, for any fixed $\varepsilon, r>0$,

$$
\begin{aligned}
\left\|\frac{g_{1} \chi_{(0, r)}}{\varepsilon V}\right\|_{\Psi_{1}(\varepsilon v)} & \leq \sup _{\int \Phi_{1}(h) \varepsilon v \leq 1} \int_{0}^{r} \frac{\int_{0}^{s} k(y) d y h(s) v(s)}{V(s)} d s \\
& =\sup _{\int \Phi_{1}(h) \varepsilon v \leq 1} \int_{0}^{r} \frac{\int_{0}^{s} k(s-t) d t h(s) v(s)}{V(s)} d s \\
& =\sup _{\int \Phi_{1}(h) \varepsilon v \leq 1} \int_{0}^{r}\left(\int_{t}^{r} k(s-t) \frac{h(s) v(s)}{V(s)} d s\right) d t \\
& \leq \sup _{\int \Phi_{1}(h) \varepsilon v \leq 1} \int_{0}^{r} k(r-t)\left(\int_{t}^{+\infty} \frac{h(s) v(s)}{V(s)} d s\right) d t .
\end{aligned}
$$

Then for any $\eta<1$, we can choose a function $h$ such that $\int_{0}^{+\infty} \Phi_{1}(h(s)) \varepsilon v(s) d s \leq 1$ and

$$
\eta\left\|\frac{g_{1} \chi_{(0, r)}}{\varepsilon V}\right\|_{\Psi_{1}(\varepsilon v)} \leq \int_{0}^{r} k(r-t) f(t) d t
$$

with $f(t)=\int_{t}^{+\infty} \frac{h(x) v(x)}{V(x)} d x$. 
Let $C_{0}$ be a constant to be determined later. Then we have

$$
\begin{aligned}
& \Phi_{2}^{-1}\left(\int_{r}^{+\infty} \Phi_{2}\left(\frac{\eta a(x)}{C_{0}}\left\|\frac{g_{1} \chi_{(0, r)}}{\varepsilon V}\right\|_{\Psi_{1}(\varepsilon v)}\right) w(x) d x\right) \\
& \leq \Phi_{2}^{-1}\left(\int_{r}^{+\infty} \Phi_{2}\left(\frac{a(x)}{C_{0}} \int_{0}^{r} k(r-t) f(t) d t\right) w(x) d x\right) \\
& \leq \Phi_{2}^{-1}\left(\int_{0}^{+\infty} \Phi_{2}\left(a(x) \int_{0}^{x} k(x-t) \frac{f(t)}{C_{0}} d t\right) w(x) d x\right) .
\end{aligned}
$$

Since $f / C_{0}$ is nonincreasing, the hypothesis of the theorem shows that the right hand side is not greater than

$$
\Phi_{1}^{-1}\left(\int_{0}^{+\infty} \Phi_{1}\left(\frac{C}{C_{0}} f(x)\right) v(x) d x\right) .
$$

Now by Lemma 4, we see that

$$
\begin{array}{r}
\Phi_{1}^{-1}\left(\int_{0}^{+\infty} \Phi_{1}\left(\int_{x}^{+\infty} \frac{C h(s) v(s)}{C_{0} V(s)} d s\right) v(x) d x\right) \\
\quad \leq \Phi_{1}^{-1}\left(\int_{0}^{+\infty} \Phi_{1}\left(\left(C C_{2} / C_{0}\right) h(x)\right) v(x) d x\right)
\end{array}
$$

holds for all $h \geq 0$. For $C_{0}$ sufficiently large, $C C_{2} / C_{1}<1$, so this is dominated by $\Phi_{1}^{-1}(1 / \varepsilon)$.

This proves the necessity of condition (5).

It remains to prove the necessity of conditions (3) and (4).

Let $g_{0}(s)=s$. We have

$$
\left\|\frac{g_{0} k(r-\cdot) \chi_{(0, r)}}{\varepsilon V}\right\|_{\Psi_{1}(\varepsilon v)} \leq \sup _{\int \Phi_{1}(h) \varepsilon v \leq 1} \int_{0}^{r} \frac{s k(r-s)}{V(s)} h(s) v(s) d s .
$$

Since $s=\int_{0}^{s} d t$, the right hand side is equal to

$$
\begin{aligned}
\sup _{\int \Phi_{1}(h) \varepsilon v \leq 1} & \int_{0}^{r}\left(\int_{t}^{r} \frac{k(r-s) h(s) v(s)}{V(s)} d s\right) d t \\
& \leq \sup _{\int \Phi_{1}(h) \varepsilon v \leq 1} \int_{0}^{r} k(r-t)\left(\int_{t}^{+\infty} \frac{h(s) v(s)}{V(s)} d s\right) d t .
\end{aligned}
$$

Here we have used the fact that $k(r-s) \leq k(r-t)$ for any $s \geq t \geq 0$. Now using the inequality (1) and proceeding as in the proof of the necessity of condition (5), we can prove the necessity of condition (3). Similarly, we can prove (4).

Thus we have proved the theorem.

\section{Proof of Theorem 2}

Before the proof, we state a few lemmas from Chapter 4 of [JS].

Lemma 5. Let $\Phi_{1}, \Phi_{2} \in \Delta_{2}$. Then there is a constant $C_{0}$ such that for all $\varepsilon, r>0$,

$$
\left\|\frac{\chi_{(0, r)}}{\varepsilon V^{\star}}\right\|_{\Psi_{1}(\varepsilon v)} \leq C_{0} \Phi_{1}^{-1}\left(\frac{1}{\varepsilon V^{\star}(r)}\right) .
$$


On the other hand, if $V^{\star}(0)=+\infty$, then there is a constant $C_{1}$ such that

$$
\left\|\frac{\chi_{(0, r)}}{\varepsilon V^{\star}}\right\|_{\Psi_{1}(\varepsilon v)} \geq C_{1} \Phi_{1}^{-1}\left(\frac{1}{\varepsilon V^{\star}(r)}\right)
$$

for any $\varepsilon, r>0$.

The detailed proof of the first inequality can be found in $[\mathrm{HK}]$. The second one can be proved similarly.

Lemma 6. Let $\Phi_{1}$ and its complementary function $\Psi_{1} \in \Delta_{2}$; then there is a constant $C$ such that

$$
\Phi_{1}^{-1}\left(\int_{0}^{+\infty} \Phi_{1}\left(\int_{0}^{x} \frac{g(y) v(y)}{V^{\star}(y)} d y\right) v(x) d x\right) \leq \Phi_{1}^{-1}\left(\int_{0}^{+\infty} \Phi_{1}(C g(y)) v(y) d y\right)
$$

holds for all $g \geq 0$.

The proof can be found in [HK]. In fact, by applying Proposition 1 to the Hardy operator, the inequality is valid provided there exists a constant $B$ such that

$$
\Phi_{1}^{-1}\left(\int_{r}^{+\infty} \Phi_{1}\left(\left\|\frac{\chi_{(0, r)}}{\varepsilon V^{\star}}\right\|_{\Psi_{1}(\varepsilon v)}\right) v(x) d x\right) \leq B \Phi_{1}^{-1}\left(\frac{1}{\varepsilon}\right)
$$

holds for all $\varepsilon, r>0$. Now this inequality follows from Lemma 5 easily.

Lemma 7. Let $\Phi_{1}$ and its complementary function $\Psi_{1} \in \Delta_{2}$; then there is a constant $C$ such that

$$
\Phi_{1}^{-1}\left(\int_{0}^{+\infty} \Phi_{1}\left(\frac{\int_{x}^{+\infty} g(y) v(y) d y}{V^{\star}(x)}\right) v(x) d x\right) \leq \Phi_{1}^{-1}\left(\int_{0}^{+\infty} \Phi_{1}(C g(y)) v(y) d y\right)
$$

holds for all $g \geq 0$.

Proof. Proposition 2 shows that the inequality is valid provided there is a constant $B$ such that

$$
\Phi_{1}^{-1}\left(\int_{0}^{r} \Phi_{1}\left(\frac{1}{V^{\star}}\left\|\frac{\chi_{(r,+\infty)}}{\varepsilon}\right\|_{\Psi_{1}(\varepsilon v)}\right) v(t) d t\right) \leq B \Phi_{1}^{-1}\left(\frac{1}{\varepsilon}\right)
$$

holds for any $\varepsilon, r>0$. The validity of this inequality is proved in [JS], page 54 .

Lemma 8. Let $\Phi_{1}, \Psi_{1} \in \Delta_{2}$. If (7) in Theorem 2 holds, then

$$
\Phi_{2}^{-1}\left(\int_{0}^{+\infty} \Phi_{2}\left[\frac{a(x)}{B} \widetilde{k}(x) \Phi_{1}^{-1}\left(\frac{1}{\varepsilon V^{\star}(0)}\right)\right] w(x) d x\right) \leq \Phi_{1}^{-1}\left(\frac{1}{\varepsilon}\right)
$$

holds for all $\varepsilon>0$.

Proof. Suppose $V^{\star}(0)<+\infty$; otherwise it is obvious. Since $V^{\star}(r) \leq V^{\star}(0)$ for any $r>0$, we have

$$
\Phi_{2}^{-1}\left(\int_{r}^{+\infty} \Phi_{2}\left(\frac{a(x)}{B} \Phi_{1}^{-1}\left(\frac{1}{\varepsilon V^{\star}(0)}\right) \tilde{k}(x-r)\right) w(x) d x\right) \leq \Phi_{1}^{-1}\left(\frac{1}{\varepsilon}\right) .
$$

Since the left hand side increases as $r$ tends to 0 , the lemma follows easily from the Monotone Convergence Theorem.

Now we begin to prove Theorem 2 . 
4.1 Proof of sufficiency. By Lemma 2, it is enough to prove (1) for functions $f$ of the form $f(x)=\int_{0}^{x} h$ with $h$ nonnegative and compactly supported in $(0,+\infty)$. Thus we have

$$
\begin{aligned}
K f(x) & =\int_{0}^{x} k(x-s)\left(\int_{0}^{s} h\right) d s=\int_{0}^{x} h(y) \int_{y}^{x} k(x-s) d s d y \\
& =\int_{0}^{x}\left(\int_{0}^{x-y} k(s) d s\right) h(y) V^{\star}(y) V^{\star}(y)^{-1} d y .
\end{aligned}
$$

Since $V^{\star}(y)^{-1}=V^{\star}(0)^{-1}+\int_{0}^{y} V^{\star}(t)^{-2} v(t) d t$ and $\widetilde{k}(x-y)=\int_{0}^{x-y} k(s) d s$, we get

$$
\begin{aligned}
K f(x) & \leq V^{\star}(0)^{-1} \int_{0}^{x} k(s) d s \int_{0}^{+\infty} h(y) V^{\star}(y) d y \\
& +\int_{0}^{x} \widetilde{k}(x-y) h(y) V^{\star}(y)\left(\int_{0}^{y} V^{\star}(t)^{-2} v(t) d t\right) d y \\
& \leq V^{\star}(0)^{-1} \int_{0}^{x} k(s) d s \int_{0}^{+\infty} f(s) v(s) d s \\
& +\int_{0}^{x}\left(\int_{t}^{+\infty} \widetilde{k}(x-y) h(y) V^{\star}(y) d y\right) V^{\star}(t)^{-2} v(t) d t \\
& \leq V^{\star}(0)^{-1} \int_{0}^{x} k(s) d s \int_{0}^{+\infty} f(s) v(s) d s \\
& +\int_{0}^{x} \widetilde{k}(x-t)\left(\int_{t}^{+\infty} f(s) v(s) d s\right) V^{\star}(t)^{-2} v(t) d t \\
& \equiv I_{1}+I_{2} .
\end{aligned}
$$

By Lemma 5, condition (7) implies

$$
\Phi_{2}^{-1}\left(\int_{r}^{+\infty} \Phi_{2}\left(\frac{a(x)}{B}\left\|\frac{\chi_{(0, r)}}{\varepsilon V^{\star}}\right\|_{\Psi_{1}(\varepsilon v)} \widetilde{k}(x-r)\right) w(x) d x\right) \leq \Phi_{1}^{-1}\left(\frac{1}{\varepsilon}\right) .
$$

For $I_{2}$, if we apply Proposition 1 with $k(x)$ replaced by $\widetilde{k}(x), b(x)=V^{\star}(x) / v(x)$ and $g(t)=v(t) \int_{t}^{+\infty} f(s) v(s) d s /\left(V^{\star}(t)\right)^{2}$, we see that the inequality

$$
\begin{aligned}
\Phi_{2}^{-1} & \left(\int_{0}^{+\infty} \Phi_{2}\left(a(x) \int_{0}^{x} \widetilde{k}(x-t) g(t) d t\right) w(x) d x\right) \\
& \leq \Phi_{1}^{-1}\left(\int_{0}^{+\infty} \Phi_{1}\left(C_{1} g(t) b(t)\right) v(x) d x\right)
\end{aligned}
$$

holds provided conditions (6) and (10) are valid.

Since $g(t) b(t)=\left(\int_{t}^{+\infty} f v\right) / V^{\star}$, Lemma 7 shows

$$
\Phi_{1}^{-1}\left(\int_{0}^{+\infty} \Phi_{1}\left(\frac{\int_{y}^{+\infty} f v}{V^{\star}}\right) v(y) d y\right) \leq \Phi_{1}^{-1}\left(\int_{0}^{+\infty} \Phi_{1}(C f(y)) v(y) d y\right)
$$

which completes the estimate for $I_{2}$. 
For $I_{1}$, take $\varepsilon>0$ such that $\int \Phi_{1}(f) \varepsilon v=1$. Then $\|f\|_{\Phi_{1}(\varepsilon v)}=1$ and

$$
\begin{aligned}
& \Phi_{2}^{-1}\left(\int_{0}^{+\infty} \Phi_{2}\left(a(x) \int_{0}^{x} k(s) d s V^{\star}(0)^{-1} \int_{0}^{+\infty} f(t) v(t) d t\right) w(x) d x\right) \\
& \leq \Phi_{2}^{-1}\left(\int_{0}^{+\infty} \Phi_{2}\left(2 a(x) \int_{0}^{x} k(s) d s V^{\star}(0)^{-1}\|f\|_{\Phi_{1}(\varepsilon v)}\|1 / \varepsilon\|_{\Psi_{1}(\varepsilon v)}\right) w(x) d x\right) .
\end{aligned}
$$

Since $\|1 / \varepsilon\|_{\Psi_{1}(\varepsilon v)}=1 /\left(\varepsilon \Psi_{1}^{-1}\left(1 /\left(\varepsilon V^{\star}(0)\right)\right)\right)$, the right hand side is not greater than

$$
\Phi_{2}^{-1}\left(\int_{0}^{+\infty} \Phi_{2}\left(\frac{2 a(x) \int_{0}^{x} k(s) d s}{\varepsilon V^{\star}(0) \Psi_{1}^{-1}\left(\frac{1}{\varepsilon V^{\star}(0)}\right)}\right) w(x) d x\right) .
$$

Noticing that $t \leq \Phi_{1}^{-1}(t) \Psi_{1}^{-1}(t)$ in [KR], page 230, it is bounded by

$$
\Phi_{2}^{-1}\left(\int_{0}^{+\infty} \Phi_{2}\left(2 a(x) \int_{0}^{x} k(s) d s \Phi_{1}^{-1}\left(\frac{1}{\varepsilon V^{\star}(0)}\right)\right) w(x) d x\right) .
$$

Replacing $f$ by $C_{0} f$ with some constant $C_{0}$, and using $\Phi_{1} \in \Delta_{2}$ and Lemma 8 , it is easy to see that the above estimate is bounded by

$$
\Phi_{1}^{-1}\left(\frac{1}{\varepsilon}\right) \leq \Phi_{1}^{-1}\left(\int_{0}^{+\infty} \Phi_{1}\left(C_{0} f(x)\right) v(x) d x\right)
$$

by the choice of $\varepsilon$. This completes the proof of sufficiency.

4.2 Proof of necessity. For the necessity of condition (6), fix any $r, \varepsilon>0$. Since the Orlicz norm dominates the Luxemburg norm, we have

$$
\left\|\frac{\widetilde{k}(r-\cdot) \chi_{(0, r)}}{\varepsilon V^{\star}}\right\|_{\Psi_{1}(\varepsilon v)} \leq \sup _{\int \Phi_{1}(g) \varepsilon v \leq 1} \int_{0}^{r} \int_{0}^{r-y} k(s) d s \frac{g(y) v(y)}{V^{\star}(y)} d y .
$$

For any $\eta<1$, take a function $g$ such that $\int_{0}^{+\infty} \Phi_{1}(g) \varepsilon v \leq 1$, and

$$
\begin{aligned}
\eta\left\|\frac{\widetilde{k}(r-\cdot) \chi_{(0, r)}}{\varepsilon V^{\star}}\right\|_{\Psi_{1}(\varepsilon v)} & \leq \int_{0}^{r} \int_{0}^{r-y} k(s) d s \frac{g(y) v(y)}{V^{\star}(y)} d y \\
& =\int_{0}^{r} k(r-s)\left[\int_{0}^{s} \frac{g(y) v(y)}{V^{\star}(y)} d y\right] d s .
\end{aligned}
$$

Take

$$
f_{r}(s)=\int_{0}^{s} \frac{g(y) v(y)}{C_{0} V^{\star}(y)} d y
$$

where $C_{0}$ is a constant to be determined later. Then by (1), we have

$$
\begin{aligned}
& \Phi_{2}^{-1}\left(\int_{r}^{+\infty} \Phi_{2}\left(\frac{\eta a(x)}{C_{0}}\left\|\frac{\tilde{k}(r-\cdot) \chi_{(0, r)}}{\varepsilon V^{\star}}\right\|_{\Psi_{1}(\varepsilon v)}\right) w(x) d x\right) \\
& \leq \Phi_{2}^{-1}\left(\int_{0}^{+\infty} \Phi_{2}\left(a(x) K f_{r}(x)\right) w(x) d x\right) \\
& \leq \Phi_{1}^{-1}\left(\int_{0}^{+\infty} \Phi_{1}\left(C f_{r}(s)\right) v(s) d s\right) .
\end{aligned}
$$


On taking $C_{0}$ sufficiently large and by Lemma 6 , the right hand side is bounded by

$$
\Phi_{1}^{-1}\left(\int_{0}^{+\infty} \Phi_{1}(g(y)) v(y) d y\right) \leq \Phi_{1}^{-1}\left(\frac{1}{\varepsilon}\right) .
$$

The Monotone Convergence Theorem allows us to replace $\eta$ above by its limiting value of 1 and thus condition (6) is proved.

Similarly, for fixed $r, \varepsilon>0$ and any $\eta<1$, we can choose a function $g$ with $\int_{0}^{+\infty} \Phi_{1}(g) \varepsilon v \leq 1$ and

$$
\eta\left\|\frac{\chi_{(0, r)}}{\varepsilon V^{\star}}\right\|_{\Psi_{1}(\varepsilon v)} \leq \int_{0}^{r} \frac{g(y) v(y)}{V^{\star}(y)} d y .
$$

Let $C_{0}$ be a constant to be determined later. Then

$$
\begin{aligned}
& \Phi_{2}^{-1}\left(\int_{r}^{+\infty} \Phi_{2}\left(\frac{\eta a(x)}{C_{0}}\left\|\frac{\chi_{(0, r)}}{\varepsilon V^{\star}}\right\|_{\Psi_{1}(\varepsilon v)} \widetilde{k}(x-r)\right) w(x) d x\right) \\
& \leq \Phi_{2}^{-1}\left(\int_{r}^{+\infty} \Phi_{2}\left(\frac{a(x)}{C_{0}} \int_{0}^{r} \frac{g(y) v(y)}{V^{\star}(y)} d y \widetilde{k}(x-r)\right) w(x) d x\right) \\
& \leq \Phi_{2}^{-1}\left(\int_{r}^{+\infty} \Phi_{2}\left(\frac{1}{C_{0} x} \int_{0}^{x} \widetilde{k}(x-y) \frac{g(y) v(y)}{V^{\star}(y)} d y\right) w(x) d x\right) \\
& \leq \Phi_{2}^{-1}\left(\int_{0}^{+\infty} \Phi_{2}\left(\frac{a(x)}{C_{0}} \int_{0}^{x} k(x-s)\left[\int_{0}^{s} \frac{g(y) v(y)}{V^{\star}(y)} d y\right] d s\right) w(x) d x\right) .
\end{aligned}
$$

By hypothesis (1), the right hand side is not greater than

$$
\Phi_{1}^{-1}\left(\int_{0}^{+\infty} \Phi_{1}\left(\frac{C}{C_{0}} \int_{0}^{x} \frac{g(y) v(y)}{V^{\star}(y)} d y\right) v(x) d x\right) .
$$

Again, on taking $C_{0}$ sufficiently large and using Lemma 6 , we have

$$
\Phi_{1}^{-1}\left(\int_{0}^{+\infty} \Phi_{1}(g(y)) v(y) d y\right) \leq \Phi_{1}^{-1}\left(\frac{1}{\varepsilon}\right) .
$$

Replacing $\eta$ by its limiting value of 1 as before, we have

$$
\Phi_{2}^{-1}\left(\int_{r}^{+\infty} \Phi_{2}\left(\frac{a(x)}{C_{0}}\left\|\frac{\chi_{(0, r)}}{\varepsilon V^{\star}}\right\|_{\Psi_{1}(\varepsilon v)} \widetilde{k}(x-r)\right) w(x) d x\right) \leq \Phi_{1}^{-1}\left(\frac{1}{\varepsilon}\right) .
$$

When $V^{\star}(0)=+\infty$, (7) follows from Lemma 5 easily. Otherwise, suppose $V^{\star}(0)<$ $+\infty$. For any $\delta>0$, replacing $v$ by $v+\delta / x^{2}$ in (1), (7) is still valid. Since

$$
\int_{0}^{+\infty}\left(v(x)+\delta / x^{2}\right) d x=+\infty
$$

by the above argument, we have

$$
\Phi_{2}^{-1}\left(\int_{r}^{+\infty} \Phi_{2}\left(\frac{a(x)}{B} \Phi_{1}^{-1}\left(\frac{1}{\varepsilon\left(V^{\star}(r)+\delta / r\right)}\right) \widetilde{k}(x-r)\right) w(x) d x\right) \leq \Phi_{1}^{-1}\left(\frac{1}{\varepsilon}\right) .
$$

Letting $\delta$ tend to 0 , the Monotone Convergence Theorem shows that (7) is valid. 


\section{REFERENCES}

[KR] M.A.Krasnosel'skii and Ya.B.Rutickii, Convex functions and Orlicz Spaces, "P.Noordhoff LTD.", 1961.

[ES] E.Sawyer, Boundedness of classical operators on classical Lorentz spaces, Studia Math. 96 (1990), 145-158. MR 91d:26026

[VS] V. Stepanov, The weighted Hardy's inequality for nonincreasing functions, Trans. Amer. Math. Soc. 338(1) (1993), 173-186. MR 93j:26012

[HS] H.P. Heinig and V.D. Stepanov, Weighted Hardy inequalities for increasing functions, Canad. J. Math. 45(1) (1993), 104-116. MR 93j:26011

[HK] H.P.Heinig and A. Kufner, Hardy operators of monotone functions and sequences in Orlicz spaces, J. London Math. Soc. 53 (1996), 256-270. MR 96m:26025

[JS] Jim Qile Sun, Hardy type inequalities on weighted Orlicz spaces, Ph.D Thesis, The Univ. of Western Ontario, London, Canada, 1995.

[RR] M.M. Rao and Z.D. Ren, Theory of Orlicz spaces, A series of Monographs and textbooks, vol. 146, Marcel Dekker, Inc., 1991. MR 92e:46059

Department of Mathematics, University of Western Ontario, London, Ontario, CANAdA N6A 5B7

E-mail address: jsuen@switchview.com 